\title{
Identification of the FDA-approved drug Pyrvinium as a novel inhibitor of PD-1/PD-L1 interaction
}

\author{
Elena Fattakhova ${ }^{[a]}$, Jeremy Hofer ${ }^{[b]}$, Juliette DiFlumeri ${ }^{[a]}$, Madison Cobb ${ }^{[a]}$, Timothy Dando ${ }^{[a]}$, Zachary \\ Romisher $^{[a]}$, Justin Wellington ${ }^{[a]}$, Michael Oravic ${ }^{[c]}$, Madison Radnoff ${ }^{[a]}$, Sachin P. Patil ${ }^{*[d]}$
}

[a] E. Fattakhova, J. DiFlumeri, M. Cobb, T. Dando, Z. Romisher, J. Wellington, and M. Radnoff Department of Chemical Engineering

Widener University

Chester, PA 19013 USA

[b] J. Hofer

Department of Computer Science

Widener University

Chester, PA 19013 USA

[c] M. Oravic

Department of Biomedical Engineering

Widener University

Chester, PA 19013 USA

[d] Dr. S. P. Patil

NanoBio Laboratory

Widener University

Chester, PA 19013 USA

E-mail: spatil@widener.edu

\begin{abstract}
Immune checkpoint blockade involving inhibition of the PD1/PD-L1 interaction has provided unprecedented clinical benefits in treating a variety of tumors. To date, a total of six antibodies that bind to either PD-1 or PD-L1 protein and in turn inhibit the PD-1/PD-L1 interaction have received clinical approvals. Despite being highly effective, these expensive large biotherapeutics possess several inherent pharmacokinetic limitations that can be successfully overcome through the use of low-molecular-weight inhibitors. One such promising approach involves small-molecule induced dimerization and sequestration of PD-L1, leading to effective PD1/PD-L1 inhibition. Herein, we present discovery of such potential bioactive PD-L1 dimerizers through a structure- and ligand-based screening of a focused library of approved and investigational drugs worldwide. The most promising compound Pyrvinium, an FDAapproved anthelmintic drug, showed $\mathrm{IC}_{50}$ value of $\sim 29.66 \mu \mathrm{M}$. It is noteworthy that Pyrvinium, being an approved drug, may prove especially suitable as a good starting point for further medicinal chemistry efforts, leading to design and development of even more potent structural analogs as selective PD-1/PD-L1 inhibitors. Furthermore, the adopted integrated virtual screening protocol may prove useful in screening other larger databases of lead- and druglike molecules for hit identification in the domain of small-molecule PD-1/PD-L1 inhibitors.
\end{abstract}

In contrast to the front-line cancer treatments like surgery, chemoand radiation therapy, cancer immunotherapy is a novel clinical approach that enhances patient's own immune system to treat tumors. As such, cancer immunotherapy has proven to be an attractive treatment strategy for a variety of traditionally challenging cancers, including melanoma, non-small-cell lung cancer and Hodgkin's Lymphoma among others ${ }^{[1]}$ and was awarded a Nobel Prize in Physiology or Medicine in 2018. [2]

The first successful cancer immunotherapy approach involved inhibition of cytotoxic-T-lymphocyte-associated protein 4 (CTLA-4), one of the immune checkpoint targets, through antiCTLA4 monoclonal antibody Ipilimumab approved in 2011.
Another important immune checkpoint pathway that has gained significant therapeutic traction recently is the protein-protein interaction between PD-1 (Programmed Cell Death Protein 1) and PD-L1 (Programmed Cell Death Ligand 1) proteins. The PD-1 is an immunoinhibitory receptor induced by the activated $T$ lymphocytes. The PD-1 receptor has two ligands, viz. PD-L1 and PD-L2, which are normally expressed on several different types of cells including dendritic cells, macrophages, activated B and $T$ cells, mesenchymal stem cells, and also nonhematopoietic cells in nonlymphoid organs like lungs, heart, and muscle. ${ }^{[3]}$ Notably, many tumor types are also known to overexpress PD-L1 on their cell surface, including squamous cell carcinoma of the head and neck, melanoma and carcinomas of bladder, breast, colorectum, kidney, liver, lung, ovary and pancreas. ${ }^{[4]}$ The interaction of PD-1 expressed on T-lymphocytes with the PD-L1 expressed on tumor cells leads to a chain of events generating immune tolerance within the tumor microenvironment through T-cell functional exhaustion and apoptosis. ${ }^{[5]}$ This in turn helps the cancer cells to avoid their elimination by the immune system.

Consequently, inhibition of the PD-1/PD-L1 interaction has proven to be a promising therapeutic strategy against a broad spectrum of cancers overexpressing PD-L1 on their surface. As of now, total six monoclonal antibodies (mAbs) have been approved for human clinical use, three each binding to PD-1 (Cemiplimab, Nivolumab and Pembrolizumab) and PD-L1 receptors (Atezolizumab, Avelumab and Durvalumab), leading to successful inhibition of PD-1/PD-L1 interaction. ${ }^{\left[{ }^{[6]}\right.}$ Although highly effective in some tumors, these large antibody therapies have several inherent pharmacokinetic limitations rendering them ineffective in over $85 \%$ of the patients that fail to respond to these checkpoint inhibitor mAbs. ${ }^{[7]}$

As a result, different alternative therapeutics that are much smaller than these mAbs are currently being explored to target the binding interfaces on both the PD-1 and PD-L1 receptors. These include small PD-1 protein fragments ${ }^{[8]}$ and macrocyclic peptides $^{[9,10]}$, many of which showed significantly better activity than the larger mAbs. These peptides and peptidomimetics thus 
can provide a good starting point in designing and optimizing the potent, truly small-molecule antagonists of the PD-1/PD-L1 interaction. Such small-molecule inhibitors may help in overcoming antibody-associated limitations such as lack of oral bioavailability, low tumor infiltration, adverse immune reactions with rare but lethal outcomes, and high cost. ${ }^{[11]}$

Despite their obvious benefits, however, the development of small-molecule inhibitors has proven challenging, owing to the unique nature of the PD-1/PD-L1 binding interface. Specifically, the PD-1/PD-L1 interface is a large, hydrophobic interface spanning over $\sim 1,970 \AA^{2}$ that lacks any well-defined binding pocket. ${ }^{[12]}$ On one hand, the PD-1 interface is highly plastic revealing a small binding cleft induced by the insertion of Tyr123 residue of PD-L1 protein (Supporting Figure S1) ${ }^{[12]}$, which has been explored previously using in silico screening leading to discovery of few small-molecule inhibitors with modest activity. ${ }^{[13]}$ In contrast, the PD-L1 binding interface is relatively featureless, rendering it useless for such systemic in silico screening efforts (Supporting Figure S2).

To overcome this challenge, an indigenous approach was utilized by Bristol Myers Squibb resulting in the discovery of a novel class of potent small-molecule inhibitors of PD-1/PD-L1 with $\mathrm{IC}_{50}$ values in $\mathrm{pM}-\mathrm{nM}$ range. ${ }^{[14]} \mathrm{X}$-ray crystallographic studies later revealed that these promising small-molecule ligands bind to PDL1 protein and in turn hinder its binding to PD-1 protein. Specifically, a single molecule of these compounds forms and stabilizes a dimer of two PD-L1 protein molecules, thus effectively blocking the PD-1 binding pockets on both the PD-L1 protein molecules. ${ }^{[15]}$ Following this successful approach, many new series of such PD-L1 dimerizing molecules have been reported recently in numerous patents. ${ }^{[14]}$ Despite many such patented small-molecule inhibitors, only two have reached the clinical investigation stage, viz. CA-107 from Curis/Aurigene (NCT02812875, clinicaltrials.gov) and INCB86550 from Incyte (NCT03762447, clinicaltrials.gov). Thus, small-molecule PD1/PD-L1 inhibitor approaches are new to cancer immunotherapy field, with no such drugs approved currently.

Therefore, we aimed to discover novel small-molecule PD1/PD-L1 inhibitors that may act through PD-L1 dimerization mechanism, with special emphasis on approved and investigational drugs that may provide immediate clinical potential against cancer. To achieve this, we utilized an integrated virtual screening approach incorporating both structure- and ligandbased screening methodologies combined with in vitro experimental testing of top virtual hits (Figure 1).

For structure-based screening, the ensemble docking of approved and investigational drugs ( 10,000 molecules) was carried out for analyzing their potential binding at the PD-L1 dimer interface. In this regard, several X-ray crystal structures of PD-L1 dimers are published so far with resolutions ranging from $1.70 \AA$ to $2.79 \AA$ (PDB IDs: 5N2F, 5NIU, 6R3K, 5J89, 5J8O, 5N2D, $6 \mathrm{NM} 8$ ). In the present study, we carried out molecular docking of approved and investigational drugs against all of these 7 PD-L1 dimer pockets using the AutoDock Vina algorithm. ${ }^{[16]}$ The AutoDock Vina correctly predicted binding modes of all the crystal ligands with the respective PD-L1 dimers, thus proving well-suited for our ensemble docking studies. The docking data from these 7 receptors were merged, and top 1,000 molecules with the best AutoDock Vina scores were selected for further analysis. The best-ranking docking poses of these top ranked compounds were visually inspected in the respective PD-L1 dimers to short-list compounds that mimic key ligand-receptor interactions similar to the published crystal ligands. These interactions included strong hydrophobic interactions with several amino acids lining the channel-like pocket of PD-L1 dimer, $\pi-\pi$ interactions with key amino acids like Tyr56, and possible hydrogen and halogen bonds at the channel opening among others.

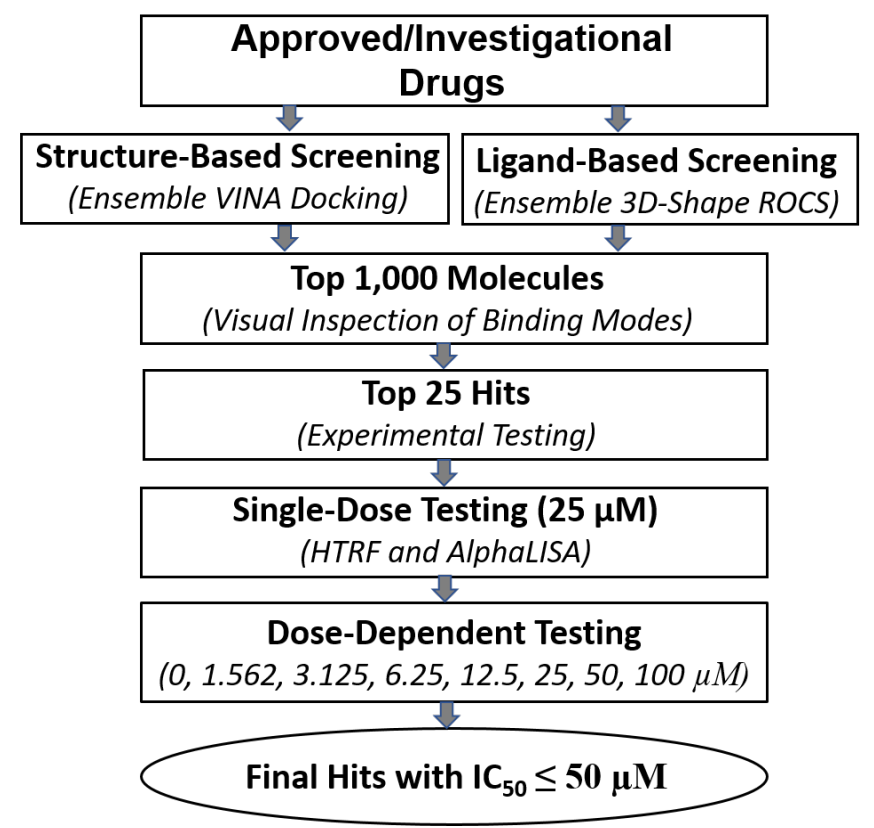

Figure 1. Integrated virtual and experimental screening workflow

The ligand-based screening was carried out using 3D shapes of 7 different crystal ligands, which present the tentative chemical and spatial requirements necessary for a potential small-molecule ligand capable of binding within the cylindrical hydrophobic pocket at the PD-L1 dimer interface. A multiconformer database of approved and investigational drugs was screened using ROCS 3.4.1.0 shape-similarity algorithm (OpenEye Scientific Software, Santa Fe, NM. http://www.eyesopen.com), to rank the drug molecules using ROCS_TanimotoCombo (shape and color) score of the highestranking conformer of each compound. The shape-similarity scoring data thus obtained using 7 crystal ligands were then combined to reveal top 1,000 molecules with the best ROCS_TanimotoCombo scores. These top ROCS hits were then docked against the high-resolution PD-L1 crystal structure (PDB: $5 \mathrm{~N} 2 \mathrm{~F}, 1.7 \AA$ ) and their binding poses were analyzed for favorable ligand-receptor interactions mentioned above.

The results from both the structure- and ligand-based screenings were then merged, resulting in a short-list of top 25 drug molecules deemed suitable for purchase based on their high docking and 3D-shape scores together with favorable binding interactions with PD-L1 dimer pocket (Supporting Table S1). These molecules were tested in two orthogonal in vitro homogenous assays (AlphaLISA and HTRF) to investigate their ability to inhibit the PD-1/PD-L1 interaction. The known PD-1/PDL1 inhibitor developed by the Bristol Myers Squibb (BMS-1166) was used as a positive control that showed dose-dependent disruption of PD-1/PD-L1 interaction with $\mathrm{IC}_{50}$ values of $0.6 \mathrm{nM}$ (HTRF) and $9.6 \mathrm{nM}$ (AlphaLISA), which are in agreement with its reported activity range of $0.06-10 \mathrm{nM}^{\left[{ }^{[17]}\right.}$ Thus, both these assays 
proved well-suited for our experimental investigations and revealed PD-1/PD-L1 inhibitory activities of these compounds to varying capacity (Supporting Table S1). As can be seen, the AlphaLISA assay showed more sensitivity, revealing a total of 4 compounds with $>40 \%$ inhibition activity at the test concentration of $25 \mu \mathrm{M}$, as compared to just one such compound revealed by HTRF assay (Table 1). It is noteworthy that compound $\mathbf{1}$ (ZINC3831401, Pyrvinium) showed comparable potency in both assays, further confirming its potential PD-1/PD-L1 inhibitory activity. Pyrvinium is an FDA-approved anthelmintic drug from the phenylpyrroles class that is used for treating pinworms.

Table 1. Top four experimentally active compounds against PD-1/PD-L1

\begin{tabular}{lllll}
\hline $\begin{array}{l}\text { Compound ID } \\
\text { (Drug Name) }\end{array}$ & $\begin{array}{l}\text { \% Inhibition } \\
\text { AlphaLISA }\end{array}$ & $\begin{array}{l}\text { \% Inhibition } \\
\text { HTRF[a] }^{[a]}\end{array}$ & $\begin{array}{l}\text { Vina } \\
\text { Score } \\
\text { (Rank) }\end{array}$ & $\begin{array}{l}\text { ROCS } \\
\text { Score } \\
\text { (Rank) }\end{array}$ \\
\hline $\begin{array}{l}\text { 1- ZINC3831401 } \\
\text { (Pyrvinium) }\end{array}$ & $44.46 \pm 3.2$ & $45.93 \pm 1.6$ & -11.0 & $\begin{array}{l}1.013 \\
(11)\end{array}$ \\
$\begin{array}{l}\text { 2- ZINC13831232 } \\
\text { (Fexaramine) }\end{array}$ & $43.32 \pm 1.1$ & $16.76 \pm 2.1$ & -11.0 & NA $^{[b]}$ \\
$\begin{array}{l}\text { 3- ZINC101331153 } \\
\text { (PF-9184) }\end{array}$ & $41.27 \pm 2.7$ & $11.66 \pm 3.6$ & -11.9 & 0.851 \\
& & & $(128)$ & $(351)$ \\
$\begin{array}{l}\text { 4- ZINC11679756 } \\
\text { (Eltrombopag) }\end{array}$ & $40.16 \pm 2.9$ & $-3.62 \pm 1.8$ & -13.3 & 0.883 \\
\hline
\end{tabular}

[a] Data are the average \pm SD of $n=3$.

[b] Compound $\mathbf{2}$ failed to generate conformers using OMEGA algorithm.

The chemical structures of these top 4 hits shown below (Figure 2) indicate that these drug molecules possess structurally novel scaffolds as compared to the known PD-1/PD-L1 inhibitors, including the crystal ligands used as templates for shapescreening. Indeed, both structure- and ligand-centric, shapebased virtual screening methodologies have been shown to lead to new inhibitors with innovative chemical scaffolds against challenging protein-protein interaction targets. ${ }^{[18,19]}$

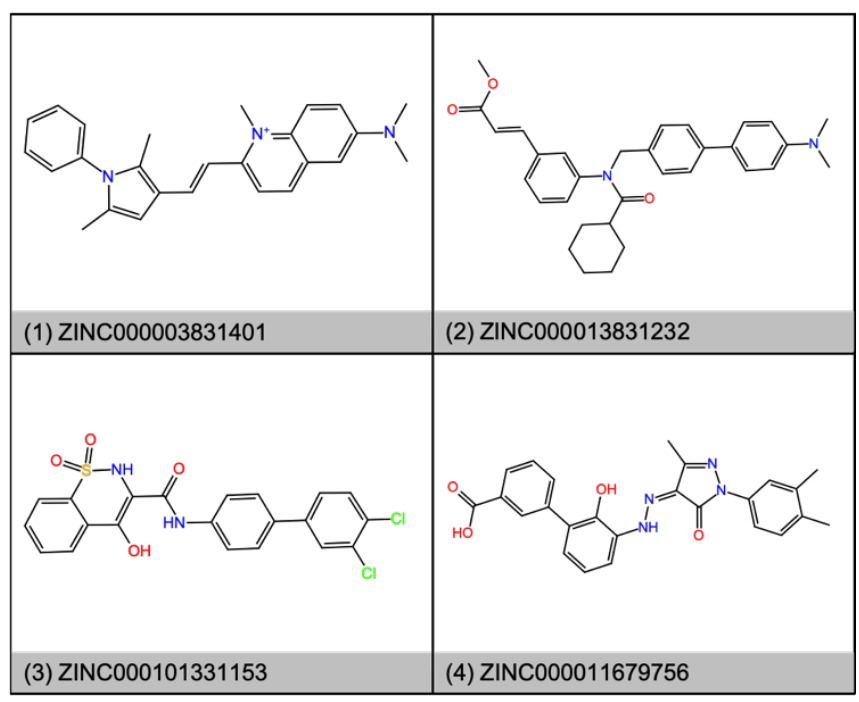

Figure 2. The 2D chemical structures of top active molecules

We further evaluated these top 4 active molecules to confirm their dose-dependent activity using both AlphaLISA and
HTRF assays. All 4 compounds showed dose-dependent activity in AlphaLISA assay with their $\mathrm{IC}_{50}$ values ranging from 25.43 43.21 $\mu \mathrm{M}$ (Figure 3). Furthermore, in line with our single-dose data, only compound 1 (ZINC3831401, Pyrvinium) showed dosedependent PD-1/PD-L1 inhibition in HTRF assay with $\mathrm{IC}_{50}$ value of $29.66 \mu \mathrm{M}$. The observed potency of Pyrvinium is remarkable considering its relatively low molecular weight (382.53 Da) and still being able to inhibit a large protein-protein interaction like PD1/PD-L1 with binding surface of $\sim 1,970 \AA^{2}{ }^{\left[{ }^{12]}\right.}$ Furthermore, Pyrvinium has the added advantage of being safe as a drug, making its use as a lead attractive.
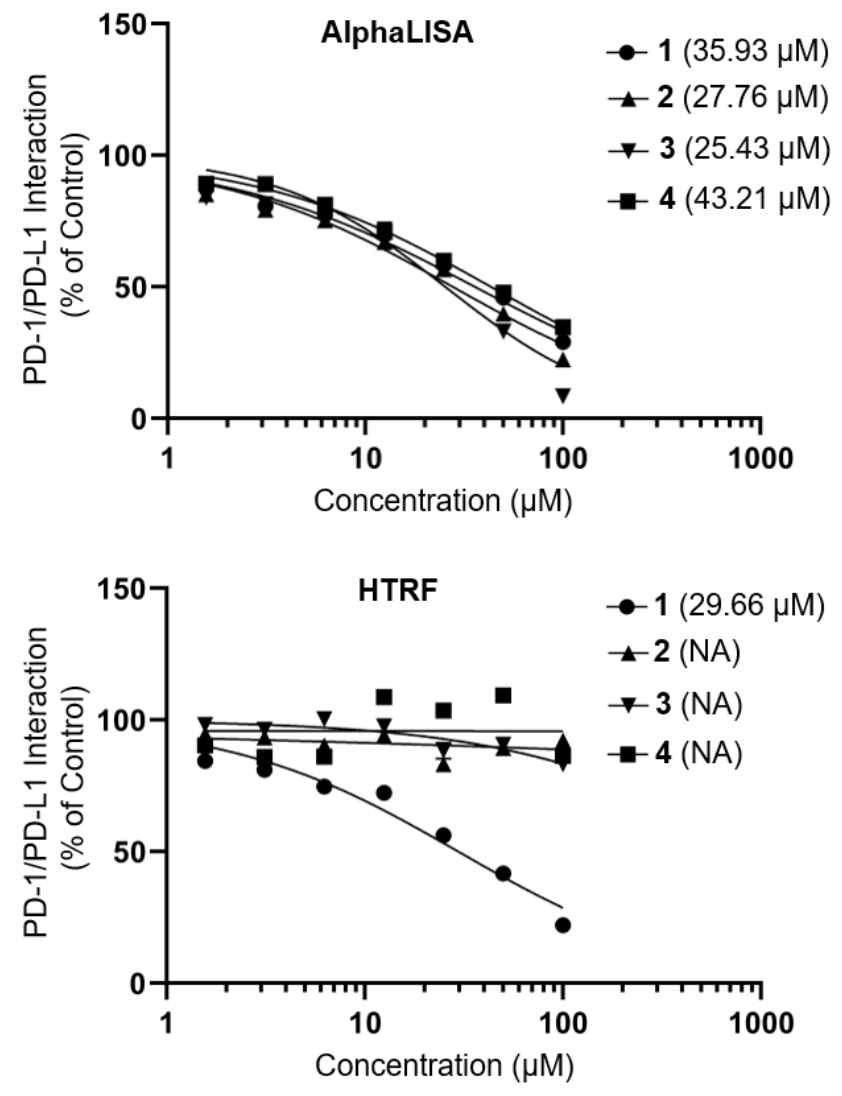

Figure 3. Dose-response curves and $\mathrm{IC}_{50}$ values for top active molecules

To study possible molecule interactions of these four active compounds within the PD-L1 dimer interface, their top-ranked Vina docking complexes with the highest resolution PD-L1 dimer structure (PDB ID: 5N2F) were individually subjected to postdocking optimizations using the default relaxation protocol in the Desmond Molecular Dynamics v3.6 package. The optimized ligand-protein complexes thus obtained revealed important interactions involved in ligand binding. The biaryl moieties in compounds 2-4 occupied the distal end of the PD-L1 dimer pocket (Supporting Figure S3) as is the case with the published PD-L1 crystal ligands. On the other hand, the dimethyl-phenylpyrrole moiety in compound $\mathbf{1}$ functionally replaced the biaryl moiety present in other three compounds and PD-L1 crystal ligands (Figure 4). The potential replacement of one or both of the methyl groups in compound $\mathbf{1}$ with bromine may lead to significant enhancement in its potency, as has been demonstrated previously with such bromine substitution leading to identification of potent compounds with low pM activity. ${ }^{[14]}$ Furthermore, 
compound $\mathbf{1}$ is predicted to make strong hydrophobic interactions with several amino acid residues lining the PD-L1 dimer channel pocket (Figure 4), while compounds 2-4 are expected to make additional hydrogen bonds especially in their solvent-exposed end (Supporting Figure S3). Thus, compound 1 may also be imparted with such $\mathrm{H}$-bonding capabilities in its solvent-exposed end, leading to further increase in the potency of such newly designed molecules. Therefore, compound 1 may prove to be an ideal scaffold for lead optimization through focused medicinal chemistry efforts.
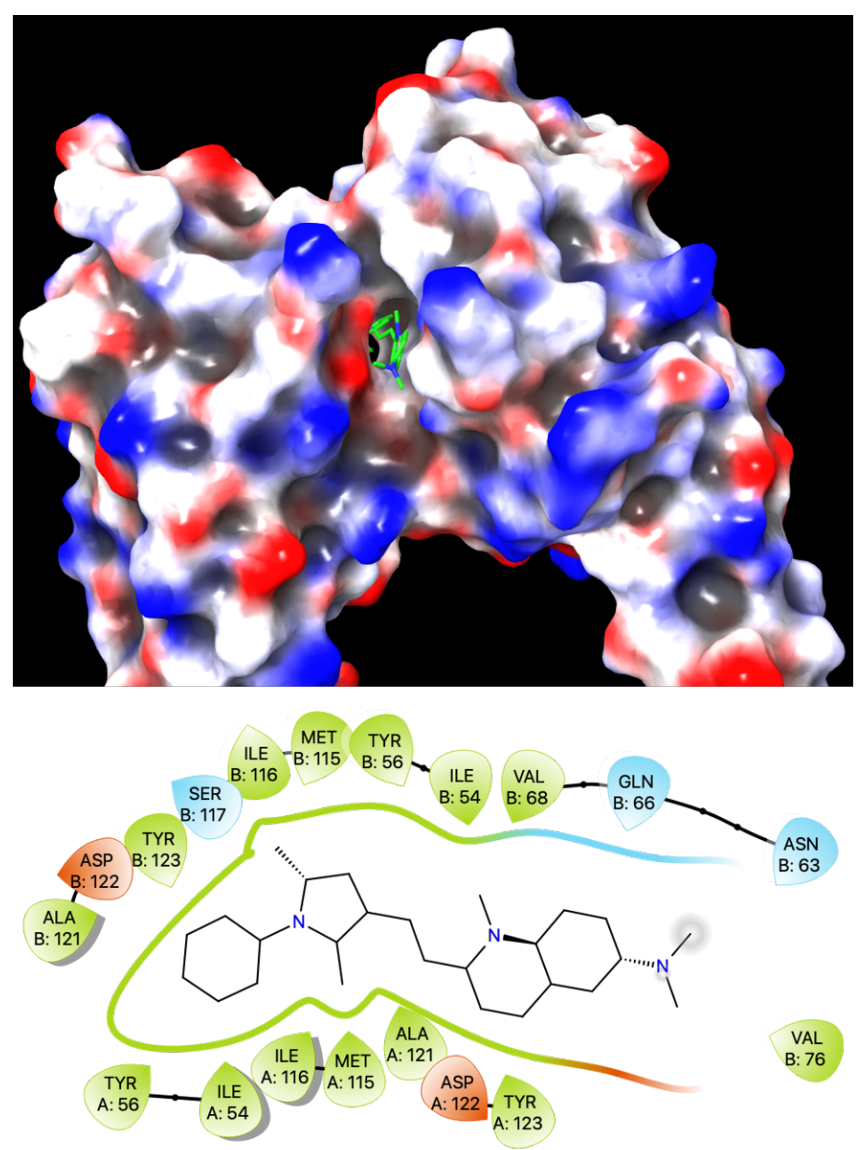

Figure 4. Predicted binding of compound 1 (Pyrvinium) within the PD-L1 dimer

In summary, our present data indicate Pyrvinium as a potential inhibitor of the immune checkpoint PD-1/PD-L1 interaction. Pyrvinium is an approved anthelmintic drug with a long history of human clinical use and thus may present an immediate clinical potential against several types of cancer expressing PD-L1 on their cell surface. Previously, Pyrvinium has been shown to exhibit anticancer activity through multiple mechanisms such as energy deprivation, Wnt suppression and anti-cancer stem cell activity. ${ }^{[20]}$ This study, however, is the first to show potential role of Pyrvinium as a small-molecule immune checkpoint inhibitor, further warranting its characterization in other suitable in vitro as well as in vivo cancer models. In addition, Pyrvinium could also serve as a promising, structurally-novel lead molecule for the development of more potent and selective, smallmolecule PD-1/PD-L1 antagonists against a variety of cancers. Finally, these promising results demonstrate potential viability of the adopted integrated virtual and experimental screening protocol for exploring other larger databases of lead- and druglike compounds for identification of potentially novel PD-1/PD-L1 antagonists for cancer immunotherapy.

\section{Experimental Section}

\section{A. AutoDock Vina Docking}

The drug database for docking was extracted from ZINC15 online repository [21], which included $\sim 10,000$ approved and investigational drugs from major jurisdictions worldwide. The ensemble docking of this drug database was carried out using 7 different crystal structures of PD-L1 dimers (PDB IDs: 5N2F, 5NIU, 6R3K, 5J89, 5J8O, 5N2D, 6NM8) downloaded from the protein data bank $(\mathrm{PDB})^{[22]}$ and processed using AutoDock Tools. ${ }^{[23]}$ The AutoDock Vina docking algorithm ${ }^{[16]}$ was used to carry out structure-based docking of the drug molecules to the PD-L1 dimer interfaces. The search space coordinates used for docking - Center:

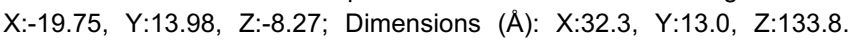
Default docking parameters were used, and the drug molecules were ranked according to their best docking score values. The AutoDock Vina data across 7 receptors were then merged, and top 1,000 molecules with best docking scores were selected for further visual inspections and selection for experimental testing.

\section{B. 3D-Shape ROCS Screening}

The ligand-based virtual screening was performed using ROCS 3.4.1.0 (OpenEye Scientific Software, Santa Fe, NM. http://www.eyesopen.com), which aligns and ranks database molecules based on the 3D shape of a given query molecule. ${ }^{[24]}$ The database of $\sim 10,000$ molecules containing approved and investigational drugs was processed using OMEGA 4.1.0.0[25] (OpenEye Scientific Software, Santa Fe, NM. http://www.eyesopen.com) using default parameters, which generated $\sim 2$ million 3D conformers. The ROCS was used to explore this $3 \mathrm{D}$ conformer database for molecules with similar shape and color as the 7 known PD-1/PD-L1 crystal ligands (crystal ligand IDs: 8HW, 8YZ, JQT, $6 \mathrm{GX}, 6 \mathrm{GZ}, 8 \mathrm{~J} 8, \mathrm{KSD})$. The database compounds were ranked by the TanimotoCombo scores for their highest-ranking conformers. The 3D shape-similarity scoring data thus obtained across 7 crystal ligands were then combined to short-list top 1,000 molecules with best TanimotoCombo scores. These top ROCS hits were then further subjected to docking against the high-resolution PD-L1 crystal structure (PDB: 5N2F, 1.7 $\AA$ ).

\section{Molecular Dynamics Methodology}

To explore possible molecular interactions of top 4 active compounds (ZINC3831401, ZINC13831232, ZINC101331153, ZINC11679756) with the PD-L1 dimer interface, molecular dynamics (MD) simulations of the respective ligand-protein complex were carried out using Desmond Molecular Dynamics package (Desmond Molecular Dynamics System, version 3.6, D. E. Shaw Research, New York, NY, 2016). The TIP3P water model was used to solvate the ligand-protein docking complexes in an orthorhombic boundary box, which were then neutralized using appropriate number of counter ions. The generated systems were equilibrated using the default relaxation protocol in the Desmond Molecular Dynamics package. The relaxation protocol included a two-step minimization (restrained and unrestrained) followed by four stages of short molecular dynamics runs with gradually diminishing restraints and increasing temperature.

\section{AlphaLISA and HTRF PD-1/PD-L1 Binding Assays}

The potential inhibition of PD-1/PD-L1 interaction by computationally selected molecules was investigated using the orthogonal AlphaLISA and HTRF assays from Perkin Elmer. The assays were carried out according to manufacturer's instructions and the assays mixtures were 
analyzed using the Alpha and HTRF protocols in BioTek Synergy $\mathrm{Neo}^{\mathrm{TM}}$ microplate reader. The percent inhibition of PD-1/PD-L1 interaction was calculated in comparison with the assay signal for the control without inhibitors (up to $1 \% \mathrm{DMSO}$ ). The assay mixture with only PD-L1 protein but not PD-1 protein indicated negative control (0\% PD-1/PD-L1 interaction). Top four active molecules exhibiting $>40 \%$ inhibition at $25 \mu \mathrm{M}$ test concentration were further subjected to dose-response experiments using both the AlphaLISA and HTRF assays. The data were analyzed using GraphPad Prism to determine $\mathrm{IC}_{50}$ values using non-linear regression variable slope models.

\section{Acknowledgements}

We thank Dr. Jeffrey Rufinus and John Stoddart (Department of Computer Science, Widener University) for use of the computer cluster. We gratefully acknowledge the support of NVIDIA Corporation with the donation of the Titan X Pascal GPU used to conduct the Desmond simulations. This work was supported by the Cancer Research Grant from W. W. Smith Charitable Trust.

\section{References:}

[1] A. Constantinidou, C. Alifieris, D. T. Trafalis, Pharmacol Ther. 2019, 194, 84-106.

[2] H. Ledford, H. Else, M. Warren, Nature 2018, 562, 20-21.

[3] W. Qin, L. Hu, X. Zhang, S. Jiang, J. Li, Z. Zhang, X. Wang, Front Immunol. 2019, 10, 2298

[4] Y. Wu, W. Chen, Z. P. Xu, W. Gu, Front Immunol. 2019, 10, 2022

[5] Y. Han, D. Liu, L. Li, Am. J. Cancer Res. 2020, 10, 727-742.

[6] A. Akinleye, Z. Rasool, J. Hematol. Oncol. 2019, 12, 92.

[7] A. Haslam, V. Prasad, JAMA Netw Open. 2019, 2, e192535.

[8] R. L. Maute, S. R. Gordon, A. T. Mayer, M. N. McCracken, A. Natarajan, N. G. Ring, R. Kimura, J. M. Tsai, A. Manglik, A. C. Kruse, S. S. Gambhir I. L. Weissman, A. M. Ring, Proc Natl Acad Sci U S A. 2015, 112, E6506E6514.

[9] K. Magiera-Mularz, L. Skalniak, K. M. Zak, B. Musielak, E. RudzinskaSzostak, Ł. Berlicki, J. Kocik, P. Grudnik, D. Sala, T. Zarganes-Tzitzikas, S. Shaabani, A. Dömling, G. Dubin, T. A. Holak, Angew. Chem. Int. Ed. Engl. 2017, 56, 13732-13735.

[10] S. P. Patil, S. C. Yoon, A. G. Aradhya, J. Hofer, M. A. Fink, E. S. Enley, J. E. Fisher, M. C. Herb, A. Klingos, J. T. Proulx, M. T. Fedorky, Chem. Pharm. Bull. 2018, 66, 773-778

[11] B. A. Baldo, Oncoimmunology 2013, 2, e26333.

[12] K. M. Zak, R. Kitel, S. Przetocka, P. Golik, K. Guzik, B. Musielak, A. Domling, G. Dubin, T. A. Holak, Structure 2015, 23, 2341-2348.

[13] S. P. Patil, M. A. Fink, E. S. Enley, J. E. Fisher, M. C. Herb, A. Klingos, J. T. Proulx, M. T. Fedorky, ChemistrySelect 2018, 3, 2185-2189.

[14] K. Guzik, M. Tomala, D. Muszak, M. Konieczny, A. Hec, U. Błaszkiewicz, M. Pustuła, R. Butera, A. Dömling, T. A. Holak, Molecules 2019, 24, 2071

[15] K. M. Zak, P. Grudnik, K. Guzik, B. J. Zieba, B. Musielak, A. Domling, G. Dubin, T. A. Holak, Oncotarget 2016, 7, 30323-30335.

[16] O. Trott, A. J. Olson, J. Comput. Chem. 2010, 31, 455-461.

[17] L. S. Chupak, M. Ding, S. W. Martin, X. Zheng, P. Hewawasam, T. P. Connolly, N. Xu, K. S. Yeung, J. Zhu, D. R. Langley, D. J. TENNEY, P M. Scola, 2015, WO Patent 2015160641 A2.

[18] S. P. Patil, P. J. Ballester, C. R. Kerezsi, J. Comput. Aided Mol. Des. 2014, 28, 89-97.

[19] S. P. Patil, M. F. Pacitti, K. S. Gilroy, J. C. Ruggiero, J. D. Griffin, J. J. Butera, J. M. Notarfrancesco, S. Tran, J. W. Stoddart, J. Comput. Aided Mol. Des. 2015, 29, 155-163.

[20] A. A. Momtazi-Borojeni, E. Abdollahi, F. Ghasemi, M. Caraglia, A. Sahebkar, J. Cell Physiol. 2018, 233, 2871-2881.

[21] T. Sterling, J. J. Irwin, J. Chem. Inf. Model. 2015, 55, 2324-2337.

[22] H. M. Berman, J. Westbrook, Z. Feng, G. Gilliland, T. N. Bhat, H. Weissig, I. N. Shindyalov, P. E. Bourne, Nucleic Acids Research 2000, 28, 235242
[23] G. M. Morris, R. Huey, W. Lindstrom, M. F. Sanner, R. K. Belew, D. S. Goodsell, A. J. Olson, J. Comput. Chem. 2009, 30, 2785-2791.

[24] P. C. D. Hawkins, A. G. Skillman, A. Nicholls, J. Med. Chem. 2007, 50, 74-82.

[25] P. C. D. Hawkins, A. G. Skillman, G. L. Warren, B. A. Ellingson, M. T. Stahl, J. Chem. Inf. Model. 2010, 50, 572-584. 